\title{
Methods for De-novo Genome Assembly
}

\author{
Arash Bayat*1,3 ${ }^{*}$ Hasindu Gamaarachchi ${ }^{1}$, Nandan P Deshpande ${ }^{2}$, Marc R Wilkins ${ }^{2}$, and Sri \\ Parameswaran $^{1}$ \\ ${ }^{1}$ School of Computer Science and Engineering, UNSW, Australia \\ ${ }^{2}$ Systems Biology Initiative, School of Biotechnology \\ and Biomolecular Sciences, UNSW, Australia \\ ${ }^{3}$ Health and Biosecurity, CSIRO, Australia
}

June 25, 2020

\begin{abstract}
Despite advances in algorithms and computational platforms, de-novo genome assembly remains a challenging process. Due to the constant innovation in sequencing technologies (Sanger, SOLiD, Illumina, 454, PacBio and Oxford Nanopore), genome assembly has evolved to respond to the changes in input data type. This paper includes a broad and comparative review of the most recent short-read, long-read and hybrid assembly techniques. In this review, we provide (1) an algorithmic description of the important processes in the workflow that introduces fundamental concepts and improvements; (2) a review of existing software that explains possible options for genome assembly; and (3) a comparison of the accuracy and the performance of existing methods executed on the same computer using the same processing capabilities and using the same set of real and synthetic datasets. Such evaluation allows a fair and precise comparison of accuracy in all aspects. As a result, this paper identifies both the strengths and weaknesses of each method. This comparative review is unique in providing a detailed comparison of a broad spectrum of cutting-edge algorithms and methods.
\end{abstract}

Availability: https://arashbayat.github.io/asm

\footnotetext{
*To whom correspondence should be addressed. Email: arash.bayat@csiro.au
} 


\section{Introduction}

$D N A$ is a giant molecular strand that is a chain of four small molecules. Sequencing is the process of reading DNA molecular chain into strings of A, C, Tand G where each letter (called a base) represents one of the small molecules. Due to the available technology today, the $D N A$ strand is broken into small pieces and each fragment is then sequenced separately. However, the order of DNA fragments (reads) cannot be preserved. Therefore, a process called genome assembly is used. This is the process by which the entire genome of an individual or species is obtained. The word assembly indicates that the entire genome cannot be obtained at once; rather, it must be assembled from small $D N A$ fragments. The process of de-novo assembly has to do with assembling a species' genome for the first time. The resulting genome can then be used to facilitate genome assembly of other individuals of the same species, in a process called the reference-guided assembly. (To understand this, one might think of solving a jigsaw puzzle by using its cover photo for reference.) In contrast, de-novo assembly refers to assembling the genome without having a draft genome; this is a complex and difficult problem to solve.

Based on the purpose of the assembly, there are several expectations about the accuracy of the assembled genome and the time it takes to be assembled. Furthermore, the type and volume of sequenced data play important roles in the assembly process. It is critical to choose a pipeline that best fits the available data and fulfils expectations. Only a fair comparison of assembly pipelines can be informative as it would truly reveal the strengths and weaknesses of various methods.

The assembled genome is expected to be accurate; various metrics can be used to measure the accuracy of an assembled genome [16]. The most important metrics include genome coverage and contiguity as well as base calling error rate. It is not possible to find a pipeline that maximizes the accuracy of all aspects 9. The pipeline should be chosen based on the application for which the genome is assembled.

If the assembled genome is going to be used as a reference-genome in a reference-guided assembly, high genome coverage is prioritized. If part of the genome is missed in the reference-genome, all subsequent assembled genomes will lack the information on that region. However, if a slight base calling error exists in the reference-genome, a variant-calling process shows the same variation in all individuals; this variation provides evidence of the error in the reference-genome. This can be fixed by updating the reference-genome for further analysis. On the other hand, if the genome is assembled for functional genomic studies, high base calling accuracy in gene areas is critical since an incorrect base calling could change the predicted protein structure. High genome coverage and contiguity in all regions may not be a benefit in this scenario.

There are expectations from the assembly pipeline as well. The program should trade-off between speed and accuracy. Despite algorithmic enhancements, speed is usually obtained by applying approximations. This leads to a less accurate assembled genome, which might, for example, consider only exact-match overlaps instead of all overlaps between reads 43]. Scalability is another expectation from an assembly pipeline [24. Genome assembly requires a massive amount of processing, and a pipeline should be able to utilize all available hardware resources such as processors and memory with the highest efficiency. The program should also be able to deal with limitations. For instance, using a large amount of memory is common in de-novo assembly programs. A scalable program should be able to deal with the limited amount of available memory, with minimal impact on execution time 28 .

In addition to the above expectations, the choice of an assembly pipeline should be based on the following 'data-specific' parameters.

- The types of data that are available for assembly: It is important to compare short-read and longread assembly approaches to each other and to hybrid assembly techniques.

- Ultra-short read sequencing such as early SOLiD 38] sequencing is suitable for deBruijn graph assembly but not an OverlapLayout-Consensus (OLC) assembly approach.

- Next-Generation Sequencing (NGS) 31 machines such as Illumina 4 produce reads up to hundreds of bases (short-reads) with high accuracy. The error rate is less than $2 \%$; most errors are of the substitution type and are less frequently short Indels.

- paired-end [23] and mate-pair [50] reads are sequenced from two ends of a long DNA fragment where the distance between them is approximately known. Such information can be used to improve the quality of the assembled genome.

- PacBio [14] and Oxford Nanopore [20] are Single-Molecule Real-Time (SMRT) technologies that can sequence reads up to many thousands of bases long. However, they suffer from a high base calling error rate (up to $30 \%$ ) and can include long Indels.

- Sequencing remains an expensive process: It is important to understand how different software programs respond to low and high read coverage depth when assembling a genome.

- If only short-read or long-read are provided, the coverage depth should be considered in configuring the assembly pipeline. For high coverage data, more filtering can be applied to collect high-confidence data for more accurate assembly. On the other hand, when the coverage is low, fewer heuristics should be applied to utilise all the information in the data. For example, one should consider a more exhaustive search to find all overlaps 
between reads, not just those that are easy to capture.

- For hybrid assembly where short and long reads are used together in an assembly, coverage depth has a stronger effect on the choice of assembly pipeline. For example, if high coverage long-reads are provided, it is possible to do a long-read-only assembly [24] and use short-read for genome polishing [51. For lower long-read coverage, long-read should be corrected with short-read [17] prior to the long-read assembly. If long-read coverage is not sufficient for assembly, it is possible to use long-read to improve contiguity of a short-read assembly [2].

- For all assemblies, it should be noted that sequencing coverage depth is not fixed across the genome. Variation in coverage results in some regions having low (or no) read coverage that leads to a discontiguity in the resulting assembly. In this case, one should consider a pipeline that includes a scaffolding step.

It is important to use the same dataset, the same computer, and the same quality assessment method for evaluation of different assembly pipelines; otherwise, the comparison would be biased and inaccurate.

In our evaluation, all tools were given the same set of synthetic and real datasets with low and high sequencing coverage depth. We performed all tests on the same High Performance Computing (HPC) computer. We used a uniform, comprehensive evaluation framework (QUAST [16]) to assess all resulting assemblies. In addition to evaluating the assemblies resulting from each pipeline, we provided accurate performance measurements of execution times and peak memory usage. We also compared read corrections and genome polishing tools.

This paper complements previously published reviews. In a recent review 46, Sohn et al. compared several short-read, long-read and hybrid assembly pipelines. However, they did not perform their own analysis; rather, they used the results provided by the author of each method for the evaluation. Thus, their comparison metrics were limited to N50, which is provided by all authors. In [9], Bradnam et al. evaluated multiple assemblies submitted by several participating teams. Their main focus was on short-read assembly. Each participating team best optimised the pipeline for the given dataset. However, the error pattern varied in accordance with the sequencing technology and sample preparation (i.e., contamination). Participating teams were able to evaluate their assembly using closely related genomes prior to submission. The work in 37] is another review paper in which long-read sequenced data $($ PacBio) are not considered. Neither of these surveys includes the most recent long-read assemblers such as Canu [24].

\section{MATERIALS AND METHODS}

\subsection{Algorithms and Methods}

The input to the assembly process is a set of reads that are passed through all data preprocessing steps. There is a graph data structure in the heart of each assembly pipeline called the assembly graph. The goal of the assembly graph is to link small $D N A$ fragments to one another to build the genome. The output of the assembly graph is a set of sequences called contigs which are usually large pieces of the genome without any information about their order in the genome. In the best scenario, there would be one contig per chromosome covering the entire chromosome. However, this is an idealistic goal for current assembly pipelines, especially when dealing with large genomes. Finally, the post-processing steps would improve the assembly by ordering these contigs, filling the gaps and fixing errors. Scaffolding is the process in which the order of the contig in the genome and the distance between them is predicted. Gap filling is the process of identifying the sequence between the scaffolds.

In this section, we first describe the data preprocessing steps that take place before graph assembly. Then we briefly explain how different graph algorithms construct contigs from the given reads. Next, we describe the postprocessing steps that take place after graph assembly.

Preprocessing

Data preprocessing involves one or more of the following steps; the last step is only applicable for shortread data.

- Filtering reads is to remove those that may increase assembly error rate.

- Fixing errors in the reads to reduce the complexity of the subsequent steps and to improve assembly quality.

- Reducing the volume of data by removing redundancy.

Read Filtering: Filtering is a commonly applied preprocessing technique for short-read data. The reads with low quality scores and/or ambiguous bases [44] are removed. Furthermore, duplicate reads can be removed [44].

short-read Error Correction: Error correction of short-reads is usually performed by utilising the $k$-mer frequency [39, 22]. k-mers are short, fixed-length subsequences of length $k$ taken from a sequence. Even after performing read filtering, reads would still contain sequencing errors. Assuming 30X depth of sequencing coverage, reads are distributed over the genome evenly; considering a low error rate in short-read, each part of the genome is expected to be covered by nearly 30 identical $k$-mers. A rare (low frequency) $k$-mer could be the result of an error in a read, or it could be the result of lack of reads covering a specific region of the genome. Any $k$-mer with low frequency can be corrected by replacing it with the closest high-frequency 
$k$-mer. However, there are difficulties in this process too; for example, regions of the genome might be repetitive or similar to other regions, resulting in uneven distribution of reads mapped across the genome.

long-read Error Correction using short-read: The error correction of long-reads requires a different approach and is more computation-intensive. In the early stage of long-read sequencing technology, the rate of sequencing error was very high and assembly of long-read data was not feasible. In addition, it was not possible to correct one long-read using the information on another long-read due to the high error rate. Since shortreads were quite accurate and cheap, using them to correct errors in long-read data became the prevailing solution [1, 41, 17, 18.

Figure 1 shows the process of mapping short-reads to long-reads which is usually done by read mappers such as $B W A$. First, the entire long-read dataset must be indexed using FM-Index. Then for each short-read several subsequences (seeds) are taken and searched to find their exact matches in the long-reads. Regions with enough similarities are called candidate regions. Each short-read is then aligned to all corresponding candidate regions. Due to high error rate in long-reads, sufficient number of short-read seeds must be searched to identify possible candidate regions [17]. Figure 2 shows the consensus process in which the target sequence (long-read here) is corrected when there is enough evidence of an error based on the mapped sequences (short-reads here). The consensus operation is used in different applications but the target sequence and the mapped sequences are different for each application.

In this method of correction, short-reads are mapped to the long-reads and then a consensus process identifies and fixes the errors in the long-reads. When mapping short-reads to long-reads, a low similarity is expected compared to mapping short-reads to a referencegenome. Hence, the mapper should perform a comprehensive search in order to map a short-read to a long-read.

This error correction method can be applied iteratively as many times as required (or feasible). For example, the method proposed in [17] applies three rounds of correction.

long-read Error Correction without short-read (Finding Overlaps): As high-coverage long-read sequencing became more affordable, tools have been developed to correct errors in long-reads without the need of shortreads $[24,49]$. This approach comprises two steps: 1) finding overlaps between long-reads; and 2) correcting errors through a consensus operation.

Exact match overlaps are not expected for longreads as the error rate is high and overlaps are usually long. Thus, any region that is sufficiently long and similar between long-reads is considered to be an overlap. To find similar regions, each read must be searched in the entire read dataset. Since scanning the entire dataset for each read is not feasible, the read dataset must be preprocessed and indexed to facilitate the search process. The produced index is used to quickly find identical $k$-mers between one read and all other reads in the dataset. When there are enough identical $k$-mers to support the possibility of overlap between two reads, further processing checks whether the overlap truly exists. Finally, reads are aligned in the overlap region using a pairwise alignment algorithm.

Due to the large size of the long-read dataset, it is not practical to index all $k$-mers that exist in the entire dataset. For large genome or high coverage sequence data, the full index cannot fit in the computer memory (RAM) 11. There are two solutions to deal with this problem [28, [5]:

- Split the read dataset into $N$ parts, such that the index for each part does not exceed available memory. This solution requires $N$ times less memory but $N$ times more processing, as each read must be searched in each part of the index separately.

- Partial indexing, in which not all $k$-mers are stored in the index. This reduces the chance of identifying common $k$-mers using the index. The lack of $k$-mers in the index is compensated for by the expected long length of overlap between long-reads, which increases the chance of finding common $k$ mers in the overlap region.

When the overlaps are discovered, a consensus operation corrects the errors in each read using all other reads overlapping the current read.

Reducing Redundancy in short-read Data: In addition to the filtering and error correction discussed above, data preprocessing in some cases can also include reducing the volume of data by removing redundancy 55] or even using a subset of all data for the assembly. However, this step is only applicable for short-reads.

Assembly Graphs

There are two assembly graphs [29]: de-Bruijn graphs [54] and overlap graphs 35 .

de-Bruijn Assembly Graph: In the de-Bruijn assembly graph, reads are split into overlapping $k$-mers. Nodes of the graph represent $k$-mers. A directed edge from node $N_{a}$ to node $N_{b}$ indicates that $N_{b}$ is next to $N_{a}$ in a read. The number of nodes in the de-Bruijn graph is the total number of identical $k$-mers in the genome plus $k$-mers that do not exist in the genome but in the reads (due to errors). The weight on the edge indicates the number of times $N_{b}$ is observed next to $N_{a}$ in all reads. Thus, the weight of an edge indicates the possibility that two $k$-mers appear after each other in the DNA sequence. A path in the graph where all edges have a high weight is likely to be a part of the genome. Figure 3 illustrates an example of de-Bruijn graph assembly.

\footnotetext{
${ }^{1}$ In 53 , nearly all $k$-mers of the human genome are indexed in about $40 \mathrm{~GB}$ space. However, for $100 \mathrm{X}$ sequencing coverage depth, the read dataset is 100 times larger than the entire genome and need much larger memory which is not available.
} 


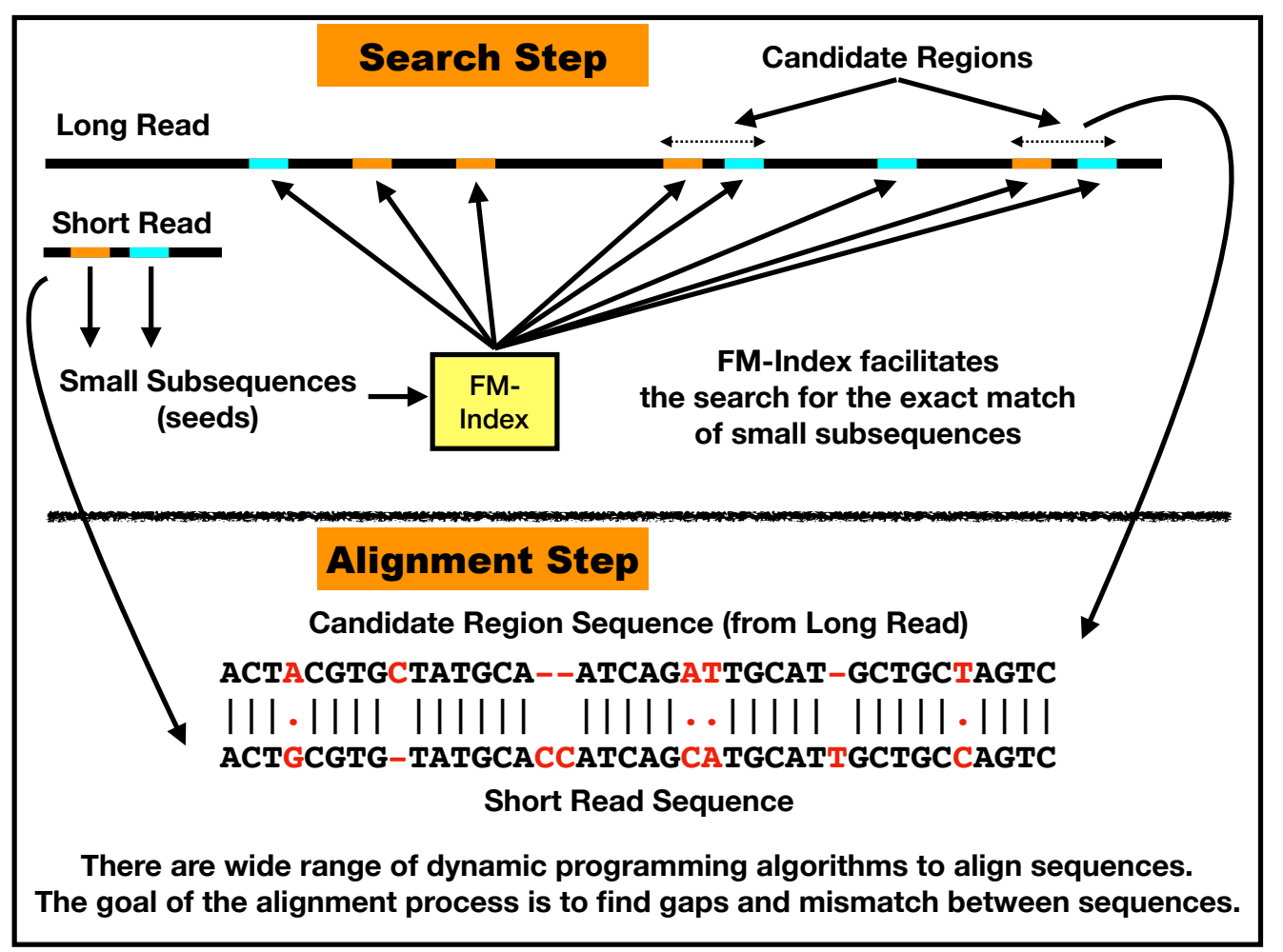

Figure 1: An example of read mapping. Subsequences of short-reads are searched in the FM-Index of long-reads. When regions with enough similarities (candidate regions) are identified, the short-read is aligned to each of those regions.

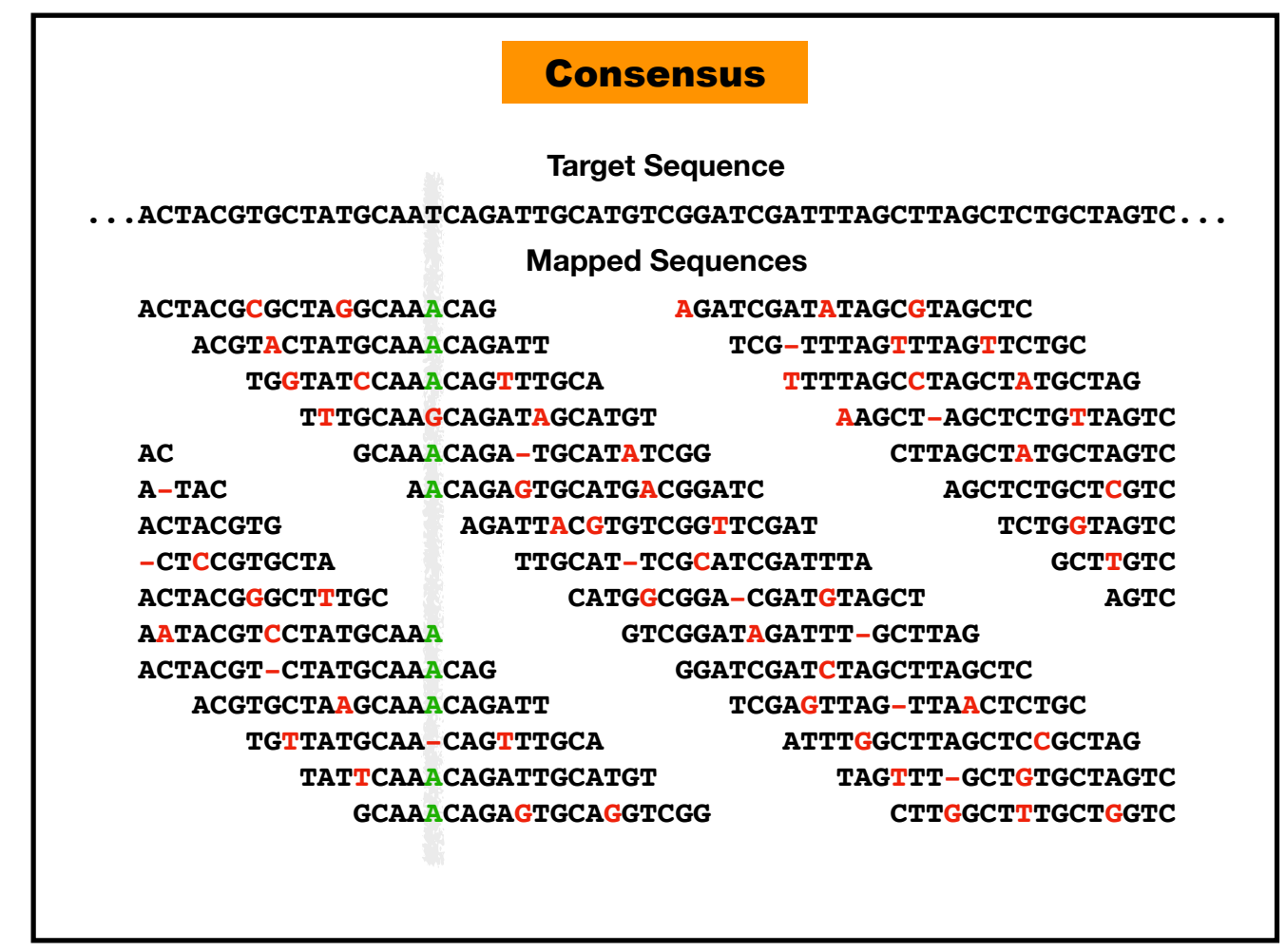

Figure 2: An example of a consensus process. The target sequence is corrected whenever there is enough evidence of error based on mapped sequences. For example the highlighted column in this figure. 
The majority of short-read assemblers use a deBruijn assembly graph. Due to the high error rate of long-reads, the de-Bruijn graph is not well suited for the long-read assembly pipeline.

OLC Assembly Graph: In Overlap-Layout-Consensus $(O L C)$ assembly (also known as string overlap graph assembly), nodes of the graph represent the entire read sequence and directed edges represent the existence of overlap between reads. For the $O L C$ graph, the number of nodes is fixed and equal to the number of input reads. In this graph, each path from node $N_{a}$ to node $N_{b}$ reveals slightly different sequences. A consensus operation constructs the most likely sequence given all paths. Figure 4 is a small example of $O L C$ based assembly.

Prior to the assembly graph traversal to produce contig sequences, the graph should be cleaned. Cleaning involves resolving loops and removing edges that may be false positives. In addition, there is a process for the string overlap graph called transitive edge reduction [42, 34] which aims to reduce redundant edges from the graph prior to the cleaning step.

The assembly graph is also able to identify the possible order of some of the contigs in the genome [27. Such information is reported along with contigs and can be utilized to increase the accuracy of the scaffolding step.

Very few short-read assemblers use $O L C$ assembly graphs. However, to the best of our knowledge, all long-read assembly pipelines use the $O L C$ approach. For the same sequencing coverage depth of the same genome, there are fewer long-reads compared to shortreads; this results in a smaller assembly graph and subsequently faster graph processing in the assembly pipeline.

\section{Postprocessing}

Postprocessing involves approaches that further improve the quality of the assembly. Three commonly used approaches are:

- Scaffolding

- Gap filling

- Genome polishing

Scaffolding and gap filling: When contigs are constructed, scaffolding [8] and gap filling [7] are two processes that join contigs to build larger contigs called scaffolds (also known as unitigs). In both scaffolding and gap filling, paired-end and/or mate-pair reads are mapped to contigs. Since the approximate distance between paired reads is known, it is possible to identify the order of contigs and the approximate distance between them in the genome. Gaps can be filled when a read is mapped to a contig and the paired read is located in the gap area. It is also possible to use longreads for scaffolding [8] and gap filling [21]. Figure 5] is an example showing scaffolding and gap filling process.

Polishing: Genome polishing is a process that can be applied to contigs and/or unitigs iteratively as many times as needed. Genome polishing fixes errors in the genome by aligning reads to the genome. If possible, utilizing data which are not employed in the assembly process would result in better polishing as the assembled genome is currently biased toward the reads used in the assembly process. In this process, additional reads are mapped to the assembled genome and then a consensus operation identifies errors. When some of the errors are fixed, there is a higher chance for reads to be mapped to the erroneous region of genome correctly; subsequently, there is a higher chance of fixing more errors [17].

Hybrid Assembly

Hybrid assembly refers to the use of multiple types of data in the assembly process. We have already pointed out some of the hybrid assembly methods above such as correction of long-read using short-read or polishing assembly using paired-end reads. However, there are several other ways in which we can utilize different types of data in the assembly process. Here we list known patterns for hybrid assembly methods. We also explain how correction and polishing processes differ from each other.

- Using short-reads to correct errors in long-reads $[1$, $1,17,41]$.

- Using short-reads to polish genomes assembled from long-reads [51].

- Using short-reads to scaffold contigs from a longread assembly pipeline.

- Using long-read to scaffold contigs from a shortread assembly pipeline [2].

- Assemble the genome with short-reads, long-reads and a hybrid pipeline, and then merge the assemblies to produce a more accurate assembly $[52$.

- Using contigs produced by short-reads to correct errors in long-reads [3].

Correction and polishing are perhaps the most common approaches in hybrid assembly pipelines. The main difference between correction and polishing is the expected error rate in the target sequence. This results in different parameters during mapping as well as different algorithms for identifying errors. In both correction and polishing, short-reads are mapped to the target sequence and a consensus process identifies and fixes the errors in the target sequence. The target sequence is usually a long-read for the correction process or a contig for the polishing process.

When mapping short-reads to long-reads, the expected similarity is low and the mapper should do a more comprehensive search in order to map a shortread to a long-read. In addition, it is less likely to map both ends of a paired-end read correctly as the mapping rate is usually low. In general, short-reads are treated as single-end reads during the long-read correction process. Mapping short-reads to contigs for polishing is a less difficult problem. In fact, one can 


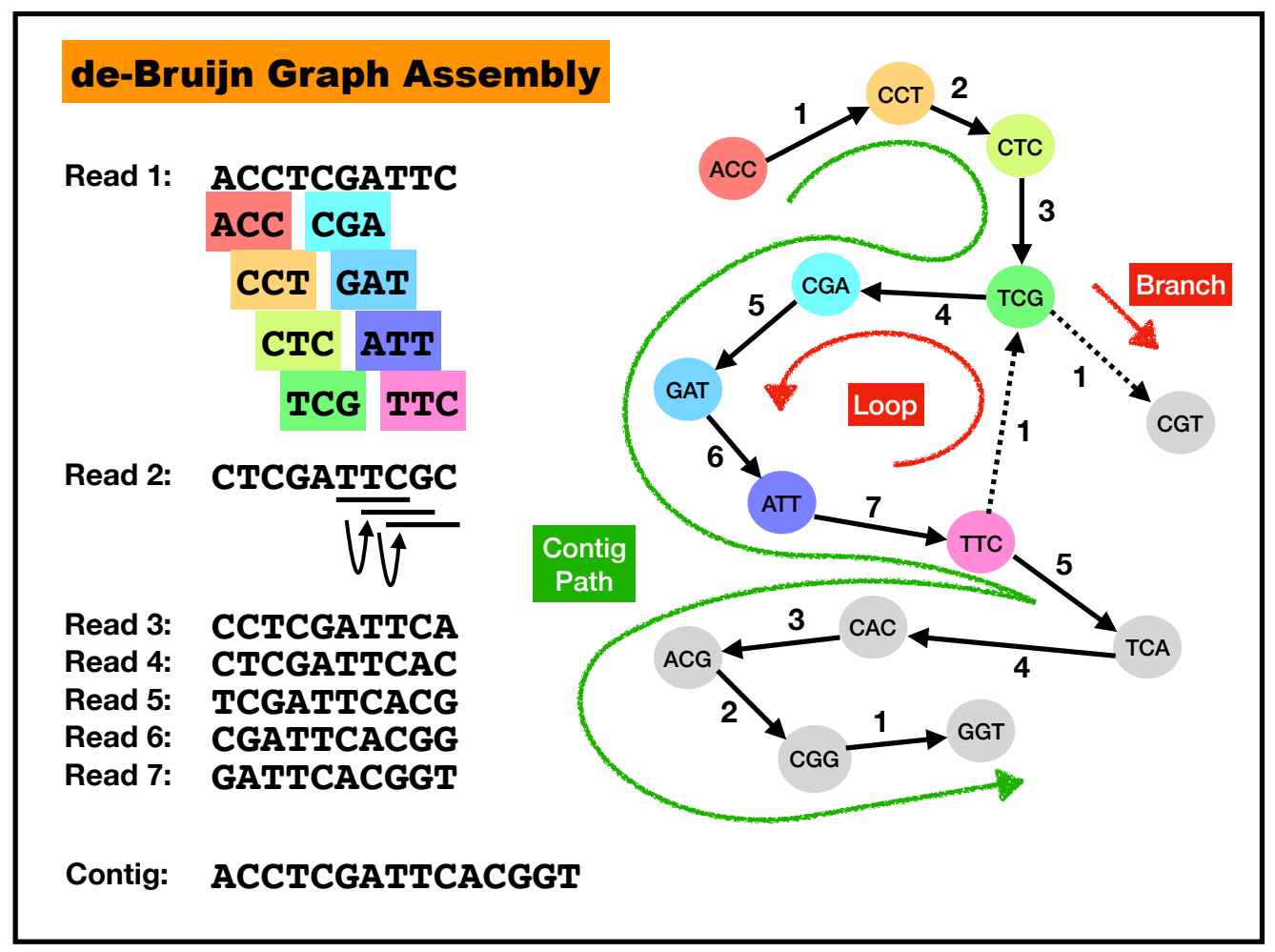

Figure 3: A tiny de-Bruijn graph example with $k$-mers of length 3. All overlapping $k$-mers taken from Read 1 are highlighted with distinct colours. The placement of these $k$-mers in the graph is also highlighted with the same colours. All k-mers form Read 2 to Read 7 are added to the graph in the same fashion. The weight on each edge of the graph represents how many times two k-mers are seen to be adjacent in the reads. The green path in the graph follows the heaviest edges and represents a contig. Due to errors in Read 2, a loop and a branch are created in the graph which is shown with the red lines. In reality, the graph is enormous with a large number of loops and many branches. 


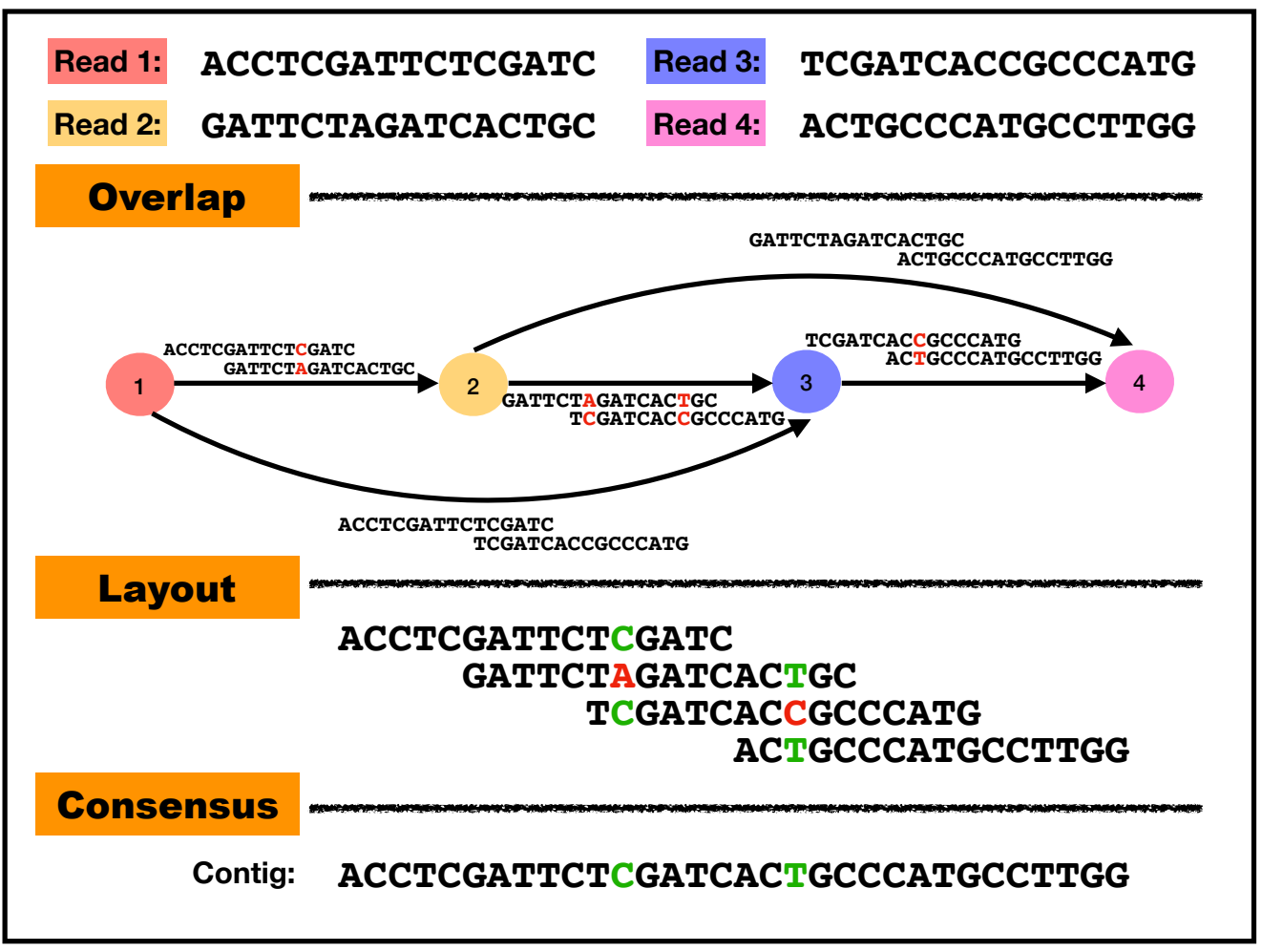

Figure 4: A tiny Overlap-Layout-Consensus graph example. In the overlap step, all overlaps between reads are listed in a graph structure. The layout step parses the graph and put the sequences in order. Finally, the consensus step generates the contig. Similar to the de-Bruijn graph, there might be loops and branches in the overlap graph which is not presented here for simplicity.

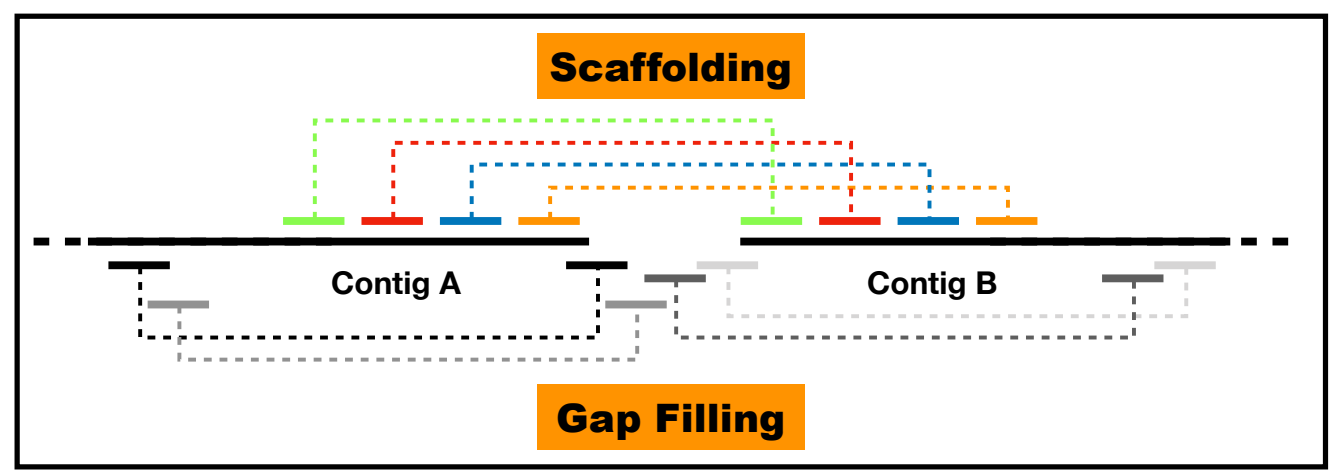

Figure 5: A small example of scaffolding and gap filling process. For the scaffolding, those paired-end and mate-pair reads are chosen that each read in a pair is mapped to the edge of a different contig. For the gap filling, one read of the pair is mapped to the edge of contig and the other is located in the gap area. 
use the same parameters as those used to map reads to the reference-genome.

For error identification, polishing tools are more conservative and require much more evidence to call an error. In addition, they are less likely to trust reads with low-quality mapping. On the other hand, the correction process tends to rely on all mapping information and is less conservative in calling errors.

Both the correction and the polishing process can be applied iteratively as many times as required (or feasible). In every iteration, more errors are corrected in the target sequence and the chance of mapping shortreads to erroneous regions is increased for the next iteration. Thus, more errors will be corrected in the next iteration. However, in reality, the number of corrected errors in each iteration drops dramatically. Furthermore, not all errors can be corrected due to difficulties in the mapping process. As a consequence, after several iterations, the number of errors remains almost constant, with slight fluctuations.

\subsection{Assembly Software and Pipelines}

short-read Assembly

To construct and traverse the assembly graph in the short-read assembly process, short-reads are usually treated as single-end reads. paired-end and mate-pair information are mainly used for scaffolding, gap filling and polishing. We elaborate three software/pipelines for short-read assembly below.

$S G A$ : String Overlap Graph $(S G A)$ [43] is a complete assembly pipeline that uses the $O L C$ approach. $S G A$ offers several tools for preprocessing and checking the quality of input data prior to assembly. Although not very common, $S G A$ also offers error correction for short-reads to improve the quality of assembly.

In order to identify overlaps between reads, $S G A$ uses FM-Index [15]. SGA limits its search to the overlaps that exactly match between reads. $S G A$ also allows searching for inexact overlaps, which have only a few differences. This increases the chance of finding more overlaps and possibly generating longer contigs. However, the associated computational cost for inexact searches are high and this option is disabled by default. Since the number of short-reads and the overlaps between them is huge, $S G A$ removes redundant overlaps using a transitive edge reduction algorithm [42] when overlaps are identified, before constructing the graph.

Since reads are randomly distributed across genomes, the chance of having an overlap of different lengths is equal. However, shorter overlaps are likely to be false-positive overlaps. As a consequence, $S G A$ only considers overlap longer than 45 base-pairs in the process. This threshold is difficult to tune as reducing it increases the number of false-positive overlaps and subsequently introduces more errors in the assembly. Conversely, the chance of having errors in longer overlaps is high and considering only exact matches results in missing many true overlaps.

There are tools in the $S G A$ pipeline for scaffolding and gap filling as well. Since $S G A$ takes a conservative assembly approach, the resulting contigs are shorter compared to Spades; instead, $S G A$ delivers the highest base calling accuracy.

Spades: Spades [2] is a de-Bruijn graph-based assembly pipeline that packs all processing steps into a single command. In addition to short-reads, Spades offers integration of other sources of data, including contigs produced by other pipelines as well as longreads. Spades uses these additional data to produce even longer contigs. Spades allows integrating longreads in a short-read assembly. long-reads are mainly used to scaffold contigs from a short-read assembly. Integration of long-reads in the Spades pipeline is discussed later in the hybrid assembly section.

SOAPdenovo2: SOAPdenovo2 [30] is an optimized de-Bruijn graph assembly pipeline that is faster than Spades and SGA. SOAPdenovo2 implements a sparse graph assembly algorithm that significantly reduces the memory footprint of the graph (the maximum memory that the program uses). It builds the graph using small $k$-mers first and corrects sequencing error. Then a large $k$-mer is used to build the graph for assembly. The larger $k$-mer better resolves loops in the graph. long-read Assembly

The advent of long-read sequencers, from PacBio and Oxford Nanopore platforms, has made it possible to assemble genomes using long reads. These may be up to tens of kilobases in length, as compared to the very short reads ( 100 bases in length) from Illumina platforms. A number of long-read assemblers have been developed to work with the long-read data.

HGAP and Falcon: HGAP [11] and Falcon [12] are early long read assembly which are widely used. HGAP uses the longest reads as seeds in a directed acyclic graphbased consensus procedure to construct a highly accurate preassembled reads. Then it follows with the assembly using off-the-shelf long-read assemblers. Falcon is another genome assembly with the focus of producing a phased diploid assembly.

Canu: One of the most recent long-read-only assembly pipelines is Canu, which was developed based on the Celera 13 assembler. Canu is a scalable software program that can automatically identify available resources and configure all parameters such that resource utilization is maximised without exceeding the available memory.

The Canu assembly pipeline uses $M H A P$ [5] to find overlaps. MHAP uses MinHash [10, which was originally developed to determine the similarity of web pages. MinHash uses a multiple hash function, which increases the chance of identifying similarities between sequences. Canu uses Myers' O(ND) algorithm 33. for the alignment and a modified version of the "falcon_sens" algorithm [12] for the correction. The Canu assembly pipeline uses the modified version of "Best Overlap Graph" 32, an $O L C$ assembly algorithm for graph assembly.

MiniC and Mini: Heng Li introduced Minimap and Miniasm in [27]. Later, the Minimap2 was introduced in [28] with several advantages compared to Minimap. Minimap2 is an overlap finding software designed for 
long-reads. Miniasm is software that can construct and traverse the assembly graph from the overlap produced by Minimap2. Since Miniasm does not have the consensus module, Racon [49] has been proposed to be used as a consensus module for Miniasm. Racon also provides the ability to correct long-read errors using long-read overlaps. However, it is claimed that Racon can assemble genomes without the need for read correction. We consider this recent long-read-only assembly pipeline using Minimap2, Miniasm and Racon in our evaluation with and without the long-read correction step. We refer these pipelines as MiniC and Mini respectively. These two pipelines are described in detail in $[28,27,49]$.

In the MiniC pipeline:

1. Minimap2 finds overlaps between long-reads.

2. Racon corrects and trims long-reads based on the discovered overlap.

3. Minimap2 finds the overlap between corrected long-reads.

4. Miniasm assembles the genome using overlaps between corrected long-reads.

5. Minimap2 finds the overlap between assembled contigs and original long-reads.

6. Racon corrects errors in the contigs.

In the Mini pipeline:

1. Minimap2 finds the overlap between long-reads.

2. Miniasm assembles the genome using the overlaps between long-reads.

3. Minimap2 finds the overlap between assembled contigs and long-reads.

\section{Racon corrects errors in the contigs.}

Although long-reads are corrected prior to assembly in the MiniC pipeline, contigs are much longer than individual long-reads; an overlap between a long-read and a contig is more reliable than the overlap between original long-reads. Thus, the contig correction in the last two steps can further improve the quality of the assembled genome.

Minimap2 uses Minimizers [40] to reduce the number of $k$-mers in the dataset to be indexed. In a window of length $k+w$ where $k$ is the length of $k$-mer, there are $w$ overlapping $k$-mers. For each window, the $k$-mer that minimizes a specific hash function is called minimizer and is stored in the index. Minimap2 stores minimizers of all overlapping windows in the input reads. Since there is a strong chance that the same $k$-mer is the minimizer of multiple overlapping windows, the number of $k$-mers to be indexed drops. However, the most efficient set of $k$-mers is selected to be indexed. In fact, MinHash used by Canu is a generalized version of Minimizer.
With smaller $w$, there would be less $k$-mer to be indexed; at the same time, however, there would be less chance to capture overlaps between reads. The small value of $k$ results in $k$-mers that are repetitive and not usable for searches. On the other hand, a large value of $k$ reduces the chance of identifying overlaps since the chance of having large identical $k$-mers is low in erroneous long-reads. An appropriate value of $k$ should be chosen with care.

Racon is error correction software for long-reads. First, the overlaps between long-reads should be discovered using Minimap2. Then the reads must be aligned in the overlap region prior to correction. Both Minimap2 and Racon can do the alignment step. Minimap2 uses the Smith-Waterman [45] algorithm implemented in K-lib [25]. Racon uses Myers' bit-vector [36] algorithm implemented in Edlib [47.

Miniasm constructs and traverses the assembly graph. Since there are false-positive overlaps, Miniasm cleans up the graph in multiple iterations. Unlike $S G A$, Miniasm removes transitive edges after constructing the graph in the memory using a modified version of the algorithm provided in [34]. Miniasm produces contig graphs, which are contigs with their possible order in the genome. However, most of the subsequent processes accept contigs in a Fasta file and do not take into account the additional information Miniasm produces. Hybrid Assembly

Colormap: Hybrid long-read error correction tools do not use $k$-mer indexing at all. Instead, they use short-read mappers such a BWA 26] and Bowtie to map short-reads to long-reads. These tools use FMIndex to index target sequences. FM-Index is a full index and its memory footprint is much smaller than $k$-mer indexing. A common FM-Index for a human genome only takes about $4 \mathrm{~GB}$ of memory. However, both index generation and search time is slower compared to the $k$-mer indexing method.

Colormap [17] is a three-iteration correction process that uses $B W A$ to map short-reads to long-reads and two correction algorithms named spCorr and $O E A$. In the first two iterations, spCorr identifies and corrects errors. In the third iteration, $O E A$ is used for correction. The third iteration can be considered a polishing step after the first two rounds of correction.

$H A L C$ : $H A L C$ [3] is another long-read correction tool that requires already assembled contigs. It first aligns long-reads to contigs using Blasr. It then corrects errors in the long-reads but not the contigs. $H A L C$ allows inputting short-reads as well, but the input contigs are mandatory. This raises the following questions about this approach:

- If the genome is already assembled, what is the purpose of correcting long-reads?

- If the correction process is biased by the current assembly, how can the corrected long-reads enhance the quality of the current assembly?

- Only long-reads that overlap with available contigs can be corrected. Those long-reads that be- 
long to undiscovered part of the genome remain untouched.

Pilon: Pilon [51] is a set of polishing tools using short-reads, which can fix base calling errors as well as some misassemblies. We polished the contigs produced by long-read assembly pipelines including Mini and Canu. We also examined the effects of applying multiple rounds of polishing.

Metassembler: Metassembler [52] is a program that allows merging multiple de-novo assemblies in order to produce a more accurate assembly. It can be considered a hybrid assembly pipeline if assemblies with short-reads and long-reads are merged together.

\section{RESULTS AND DISCUSSION}

In order to evaluate existing de-novo assembly tools, we use three real and two synthetic datasets. Real datasets are sequenced data along with high quality assembled genome from three strains of Klebsiella pneumoniae provided by Bioplatforms Australia [6]. For each real dataset, the provided high-quality assembled genome is used as a ground truth genome. More details on the real datasets we used are available in [6]. There are considerations regarding the use of both real and synthetic datasets.

- Synthetic datasets do not reflect the full complexity existing in a real dataset. For example, a real dataset includes contamination and structural error where simulators can rarely model them accurately. However, for an accurate comparison, the ground truth genome is required, especially when the error rate of evaluated assembly pipelines is low. Using a high-quality assembled genome (in a real dataset) as a ground truth might not distinguish accurate pipelines precisely.

- The complexity that exists in real datasets greatly depends on the sample preparation method (including contamination and sample quality) and sequencing technology (the type of errors). This complexity can vary between real datasets. Thus, evaluation based on one real dataset may not be the same as the comparison based on another real dataset.

- Real datasets used in this evaluation are limited to a small bacteria genome. Using a synthetic dataset, we are able to evaluate existing assembly methods when dealing with larger genomes.

For each genome, we prepared short-reads and longreads with high and low read coverage depth (40X and $20 \mathrm{X}$ respectively) to evaluate the effect of read coverage on assembly pipelines. For the synthetic dataset, we used ART [19] to simulate short-reads and SimLoRD [48] to simulate long-reads. Table 1 represents datasets used in our evaluation. The smaller synthetic genome (SIM5M) was generated by taking the first five million base-pairs of the reference-genome used for AJ055 evaluation. The larger synthetic genome (SIM25M) was 25 million base-pairs taken from part of human reference-genome (hg19) chromosome 1 (excluding $\mathrm{N}$ in the sequence). We were not able to process the larger genome as some pipelines exceeded our computational resources to process data. All real and synthetic short-reads were of length 150 base-pair.

All of our tests were run on a Linux machine with 16 processors and $64 \mathrm{~GB}$ of memory. Except for Canu, which automatically identifies and utilizes resources, we set the parallelisation factor to 16 for all tools where applicable. In all our evaluations, we ensured there was sufficient RAM in the system and neither of our tests reached system swap memory. If this happens for a process, it will noticeably slow down the process and the measured execution time would not be appropriate for comparison.

In the rest of this section, we compare a number of de-novo genome assembly pipelines using the datasets and machine described above. To ensure a fair comparison across the assembly pipelines, there are some important considerations which are also highlighted in [9].

- We did not observe one pipeline to be best in all aspects. One pipeline was fast, one was scalable, one delivered the highest base calling accuracy, one resulted in the highest genome coverage, and one produced the longest contigs.

- The application for which the genome is assembled defines which pipeline is the most appropriate. In order to compare assembly pipelines, one should know what the expectations are regarding the accuracy of the assembled genome (genome coverage, contig length, base calling accuracy) and what the expectations are regarding computational costs (scalability, speed and maximum memory usage).

- Each pipeline accepts several parameters that can significantly affect its performance and accuracy. In our evaluation, we mostly ran pipelines with their default parameters unless otherwise mentioned. If the same dataset is processed with the same pipeline but different parameters, it is possible to get different speeds and accuracy.

The runtime and the memory usage for all pipeline processing all dataset are provided in Figure6 and Figure 7 respectively. We group accuracy metrics both by the dataset and by the pipeline. For example, the tabular data in Figure 8 shows accuracy metrics for dataset AJ055 processed by all pipelines and Figure 9 shows all accuracy metrics for all datasets processed by $M i n i C$ pipeline. The complete evaluated metric and the QUAST interactive report (including charts) in $H T M L$ format, along with script we used to prepare synthetic data and execute each pipeline are available on https://arashbayat.github.io/asm. Note that the evaluation results discussed in the paper are mainly related to the AJ055 dataset. Also, note that QUAST does not consider contigs shorter than 500 base-pair in the evaluation. 
Table 1: Datasets used in out evaluation. Dataset with AJ prefix are real datasets and datasets with SIM prefix are synthetic datasets.

\begin{tabular}{c|c} 
Dataset & Genome Size \\
\hline AJ055 & $\sim 5.5 \mathrm{Mbp}$ \\
AJ218 & $\sim 5.5 \mathrm{Mbp}$ \\
AJ292 & $\sim 5.5 \mathrm{Mbp}$ \\
SIM5M & $5 \mathrm{Mbp}$ \\
SIM25M & $25 \mathrm{Mbp}$
\end{tabular}

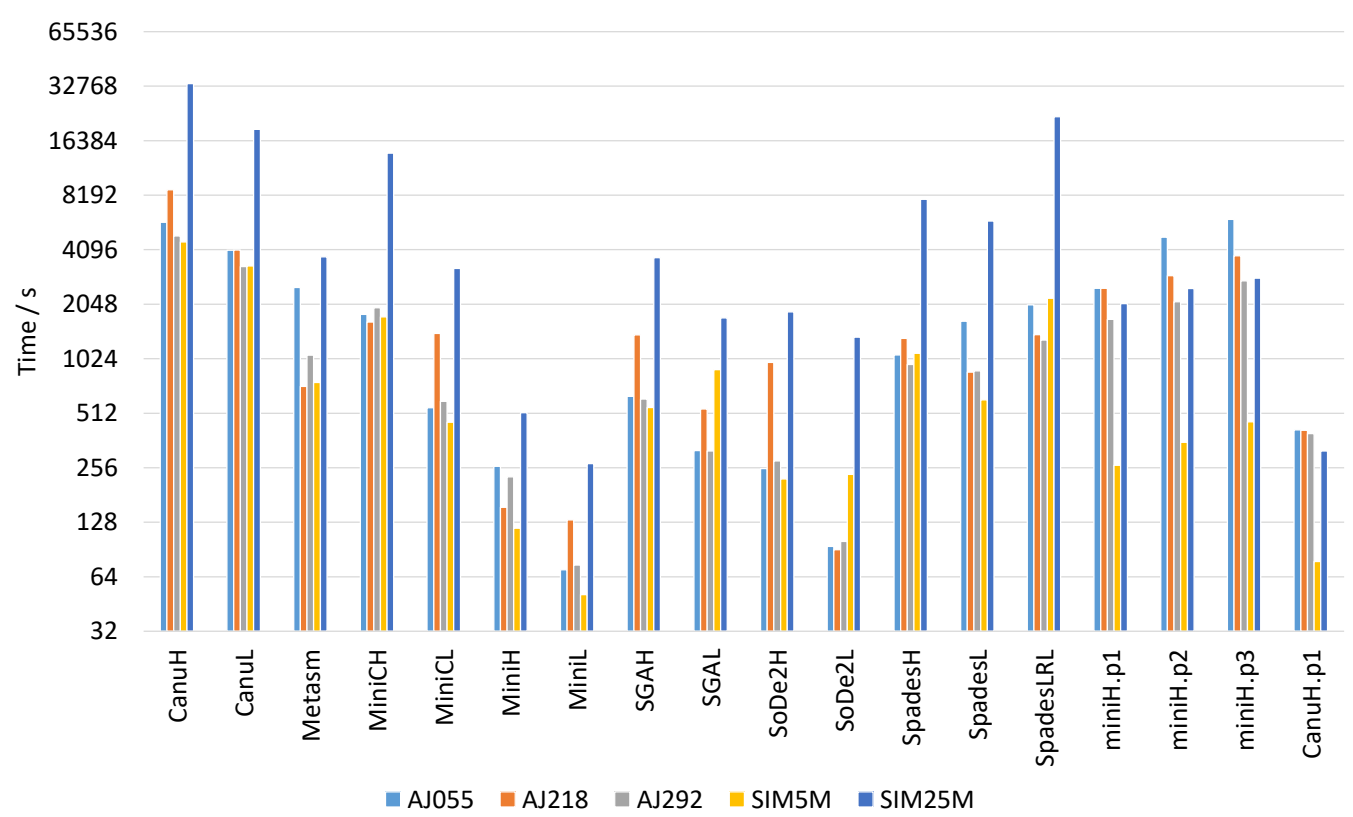

Figure 6: Colour coded runtime for all pipelines processing all datasets. For the details of assembly pipeline names refer to the first 5 rows of Figure 8. Green is better.

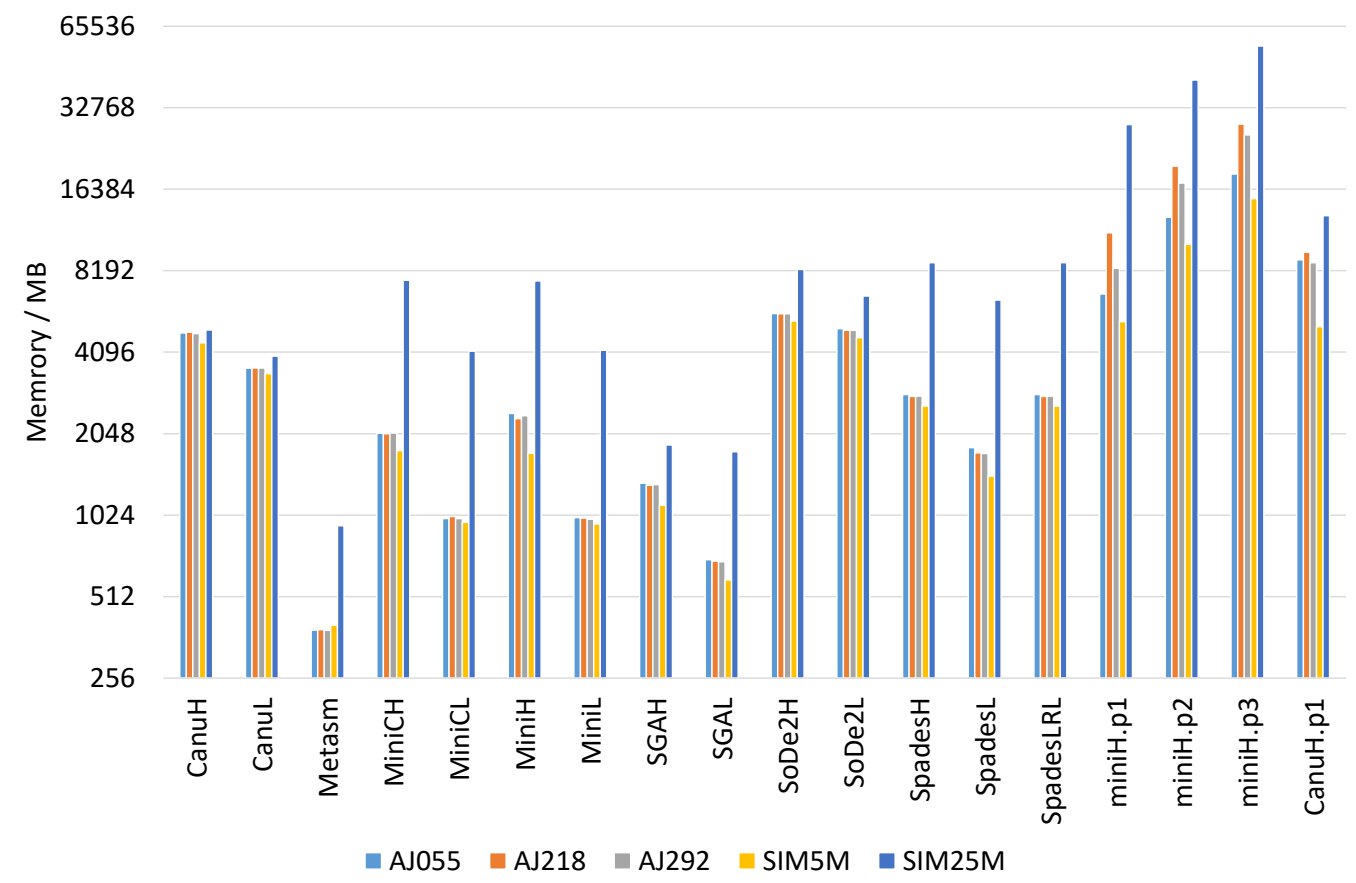

Figure 7: Colour coded Pick memory usage for all pipelines processing all datasets. For the details of assembly pipeline names refer to the first 5 rows of Figure 8. Green is better. 


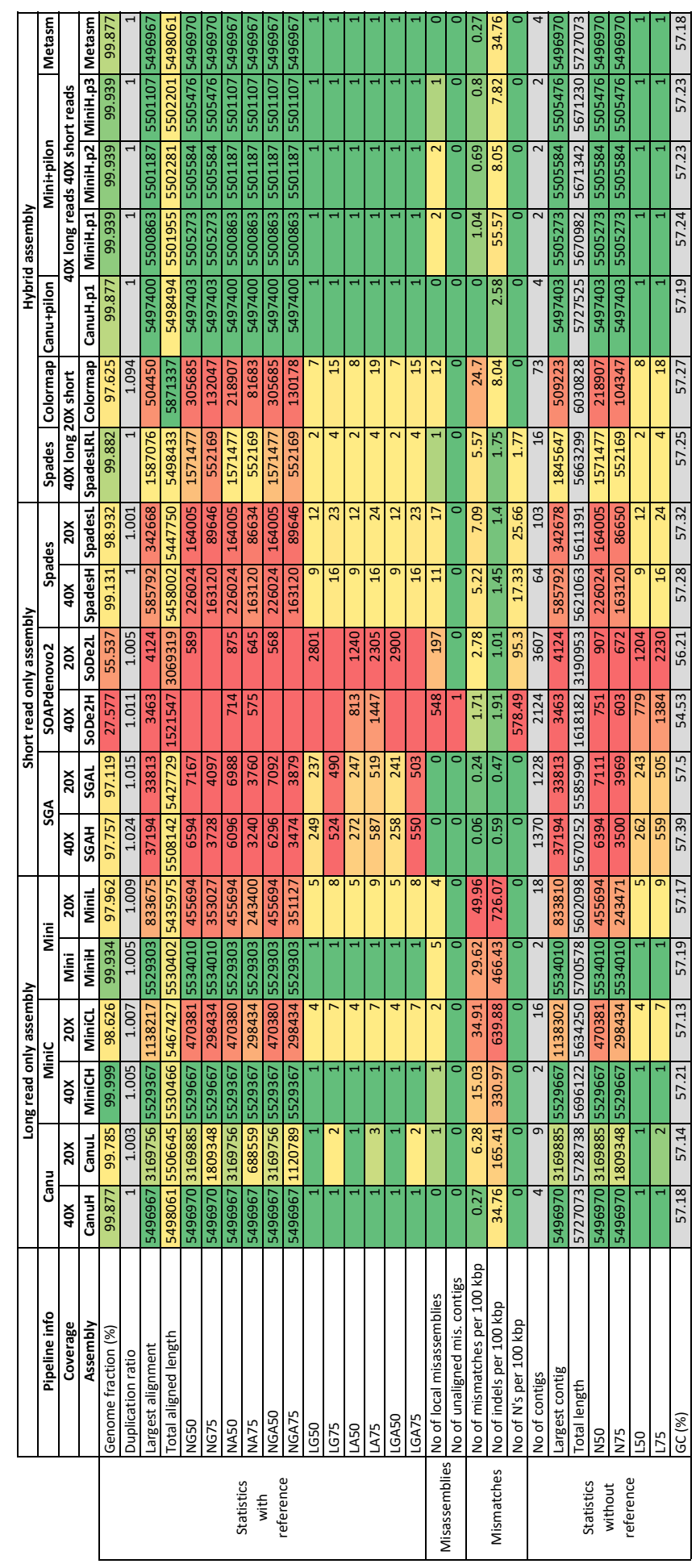

Figure 8: Accuracy metrics of all pipelines processing AJ055 dataset. All cells of the table are colour coded such that green, yellow and red represent best, moderate and worst respectively. 


\begin{tabular}{|c|c|c|c|c|c|c|c|c|c|c|}
\hline \multirow{3}{*}{\begin{tabular}{|l|} 
Assembly \\
Dataset \\
\end{tabular}} & \multicolumn{5}{|c|}{$40 \mathrm{X}$ coverage long reads } & \multicolumn{5}{|c|}{$20 \mathrm{X}$ coverage long reads } \\
\hline & \multicolumn{5}{|c|}{ MiniCH } & \multicolumn{5}{|c|}{ MiniCL } \\
\hline & AJ055 & AJ218 & AJ292 & $\operatorname{sim} 5 \mathrm{M}$ & $\operatorname{sim} 25 \mathrm{M}$ & AJ055 & AJ218 & AJ292 & $\operatorname{sim} 5 \mathrm{M}$ & $\operatorname{sim} 25 M$ \\
\hline Genome fraction (\%) & 99.999 & 1 99.977 & 99.999 & 1) 99.896 & $\begin{array}{ll}99.937 \\
\end{array}$ & 98.626 & 99.273 & 98.916 & 99.556 & 99.536 \\
\hline \begin{tabular}{|l} 
Duplication ratio \\
\end{tabular} & 1.005 & 1.006 & 1.004 & 1.007 & 1.011 & 1.007 & 1.009 & 1.005 & 1.021 & 1.012 \\
\hline Largest alignment & 5529367 & 5049176 & 5463805 & 3537045 & 4236176 & 1138217 & 1163455 & 1660035 & 1341548 & 2343337 \\
\hline Total aligned length & 5530466 & 5496411 & 5463805 & 5029349 & 25259712 & 5467427 & 5472727 & 5410760 & 5077176 & 25170834 \\
\hline NG50 & 5529667 & 5485519 & 5464361 & 3537045 & 2347005 & 470381 & 615749 & 1327492 & 704173 & 1073552 \\
\hline NG75 & 5529667 & 5485519 & 5464361 & 1235134 & 1588793 & 298434 & 459808 & 720527 & 413350 & 761653 \\
\hline NA50 & 5529367 & 5049176 & 5463805 & 3537045 & 2347005 & 470380 & 610591 & 1327480 & 704169 & 1073551 \\
\hline NA75 & 5529367 & 5049176 & 5463805 & 1235133 & 1189240 & 298434 & 459808 & 638853 & 413350 & 758216 \\
\hline NGA50 & 5529367 & 5049176 & 5463805 & 3537045 & 2347005 & 470380 & 610591 & 1327480 & 704169 & 1073551 \\
\hline NGA75 & 5529367 & 5049176 & 5463805 & 1235133 & 1189240 & 298434 & 459808 & 638853 & 413350 & 758216 \\
\hline LG50 & 1 & 1 & 1 & 1 & \begin{tabular}{l|l}
4 & 2
\end{tabular} & 4 & 3 & 2 & 3 & 8 \\
\hline LG75 & 1 & 1 & 1 & 2 & 7 & 7 & 6 & 4 & 5 & 15 \\
\hline LA50 & 1 & 1 & 1 & 1 & 4 & 4 & 3 & 2 & 3 & 8 \\
\hline LA75 & 1 & 1 & 1 & 2 & 8 & 7 & 6 & 4 & 5 & 15 \\
\hline LGA50 & 1 & 1 & 1 & 1 & 4 & 4 & 3 & 2 & 3 & 8 \\
\hline LGA75 & 1 & 1 & 1 & 2 & 8 & 7 & 6 & 4 & 5 & 15 \\
\hline No of local misassemblies & 1 & 4 & 2 & 0 & 0 & 2 & 10 & 1 & 0 & 6 \\
\hline No of unaligned mis. contigs & 0 & 1 & 0 & 0 & 이 & 0 & 1 & 0 & 0 & 0 \\
\hline No of mismatches per $100 \mathrm{kbp}$ & 15.03 & 9.44 & 9.64 & 1.28 & 2.77 & 34.91 & 20.6 & 17.79 & 5.75 & 8.92 \\
\hline No of indels per $100 \mathrm{kbp}$ & 330.97 & 248.34 & 247.97 & 165.99 & 166.48 & 639.88 & 455.2 & 409.22 & 207.88 & 198.9 \\
\hline No of N's per $100 \mathrm{kbp}$ & 0 & 0 & 0 & 0 & \begin{tabular}{l|l}
0 \\
\end{tabular} & 0 & 0 & 0 & 0 & 0 \\
\hline No of contigs & 2 & 2 & 1 & 3 & 23 & 16 & 14 & 6 & 13 & 43 \\
\hline Largest contig & 5529667 & 5485519 & 5464361 & 3537045 & 4487765 & 1138302 & 1168567 & 1660035 & 1348395 & 2343337 \\
\hline Total length & 5696122 & 5688657 & 5464361 & 5029350 & 25259994 & 5634250 & 5675913 & 5414604 & 5087756 & 25176816 \\
\hline N50 & 5529667 & 5485519 & 5464361 & 3537045 & 2347005 & 470381 & 615749 & 1327492 & 704173 & 1073552 \\
\hline N75 & 5529667 & 5485519 & 5464361 & 1235134 & 1189240 & 298434 & 459808 & 720527 & 413350 & 761653 \\
\hline$L 50$ & 1 & 1 & 1 & 1 & 4 & 4 & 3 & 2 & 3 & 8 \\
\hline L75 & 1 & 1 & 1 & 2 & 8 & 7 & 6 & 4 & 5 & 15 \\
\hline
\end{tabular}

Figure 9: Accuracy metrics of all datasets processed by MiniC pipeline. All cells of the table are colour coded such that green, yellow and red represent best, moderate and worst respectively.

\section{1 short-read Assembly}

Table 2 compares the results for three short-read assembly pipelines. SOAPdenovo2 is faster than other pipelines but results in poor assembly quality. $S O A P$ denovo2 requires more memory compared to other assembly pipelines. Spades is the slowest pipeline but results in much longer contigs and higher genome coverage compared to the others. Spades memory consumption is not as low as $S G A$ and not as high as $S O A P d e n o v o 2$. SGA results in the highest base calling accuracy and requires the lowest amount of memory. However, the contiguity of the genome assembled by $S G A$ is low.

Comparing high and low coverage data reveals an interesting point about each of these programs. In all programs and for all datasets, the processing time and peak memory usage is less for low coverage data compared to high coverage data. For $S G A$ pipelines, the execution time and peak memory usage seem to have a linear relationship with the read coverage. For SOAPdenovo2, the execution time is 2.7 times higher than for twice the amount of input, which indicates $S O A P$ denovo2 runtime is more sensitive to the number of input reads. On the other hand, Spades seems to be less sensitive to the size of input data and is only 1.6 times slower for twice as many reads. The peak memory usage of Spades and SOAPdenovo2 do not seem to be linear either.

In terms of contiguity, Spades utilized higher read coverage well and produced longer contigs. On the other hand, the evaluation shows that $S G A$ and $S O A P$ denovo2 produce longer contigs for $20 \mathrm{X}$ data compared to $40 \mathrm{X}$ data. While this is unexpected, it is true for all other genomes. Note that QUAST only considered contigs longer than 500 base-pairs for evaluation. Since there were not enough contigs longer than 500 base-pairs, the NGA50 is not computed for SOAPdenovo2 when processing 40X data. The NGA50 is the N50 for the portion of contigs that are aligned to the ground truth genome, excluding overlaps. The decrease in contiguity when a higher depth of read coverage was used could be the result of algorithmic weaknesses when dealing with a larger and more complex assembly graph.

For Spades and SGA, using 40X data resulted in a slight improvement in genome coverage compared to using 20X data. When the sequencing cost matters, one should consider whether this slight improvement is worth spending more on sequencing. The genome coverage was low for SOAPdenovo2 when processing a real dataset. Also, the genome coverage of $40 \mathrm{X}$ data was lower than genome coverage of $20 \mathrm{X}$ data. The difference is significant for real data. In addition to the weakness of SOAPdenovo2 in genome assembly, one reason for the lack of genome coverage is the fact that QUAST throws out the contigs shorter than 500 base-pairs prior to evaluation.

The error rate of all programs are generally low (being less than 3 errors per $100 \mathrm{kbp}$ ), except for mismatch errors of Spades. Note that to improve contiguity, the program should be less conservative and allow low coverage regions to be assembled as well. This can be a reason for the increase in the base calling error rate. Since the base calling accuracy was high in the shortread assembly approach, we did not study the effect of genome polishing on the resulting assembly. 
Table 2: Comparison of short-read assembly pipeline for the assembly of the AJ055 genome.

\begin{tabular}{|c|c|c|c|c|c|c|}
\hline \multirow{2}{*}{$\begin{array}{l}\text { Pipeline Name: } \\
\text { Pead Coverace \& }\end{array}$} & \multicolumn{2}{|c|}{ SGA } & \multicolumn{2}{|c|}{ SOAPdenovo2 } & \multicolumn{2}{|c|}{ Spades } \\
\hline & $40 \times \mathrm{SR}$ & $20 \times \mathrm{SR}$ & $40 \times \mathrm{SR}$ & $20 \times \mathrm{SR}$ & $40 \times \mathrm{SR}$ & $20 \times \mathrm{SR}$ \\
\hline Genome Coverage (\%) & 97.757 & 97.119 & 27.577 & 55.537 & 99.131 & 98.932 \\
\hline \# Contigs & 1370 & 1228 & 2124 & 3607 & 64 & 103 \\
\hline N50 & 6394 & 7111 & 751 & 907 & 226024 & 164005 \\
\hline NGA50 & 6296 & 7092 & & 568 & 226024 & 164005 \\
\hline \# Mismatches per $100 \mathrm{kbp}$ & 0.06 & 0.24 & 1.71 & 2.78 & 5.22 & 7.09 \\
\hline \# Indels per $100 \mathrm{kbp}$ & 0.59 & 0.47 & 1.91 & 1.01 & 1.45 & 1.4 \\
\hline \# Local Misassemblies & 0 & 0 & 548 & 197 & 11 & 17 \\
\hline Execution Time (sec) & 633 & 318 & 253 & 94 & 985 & 641 \\
\hline Memory Usage (MB) & 1376 & 717 & 5824 & 5125 & 2925 & 1859 \\
\hline
\end{tabular}

We also evaluated these assembly pipelines with a synthetic genome nearly five times longer to see the effect of genome size on the speed and accuracy of the assembly. The evaluation result for the longer genome (SIM25M). The evaluation showed that $S G A$ genome coverage declined to about $90 \%$ for the longer genome, while the Spades genome coverage remained above $98 \%$. The execution time and peak memory usage were also affected by the genome size, as shown in Table 3. While the execution time is linearly increased with the genome size for the SGA, SOAPdenovo2 and Spades show a higher sensitivity to the genome size. The genome size had less effect on peak memory usage and its effect varied between tools and differing read coverage.

\section{$3.2 \quad$ long-read Assembly}

\section{Error Correction}

In order to distinguish between different correction algorithms, we corrected both low coverage (20X) and high coverage (40X) long-read datasets using a Canu correction module and a combination of Minimap2 and Racon (alignment was done by Racon). We evaluated the corrected read using QUAST. Since the entire set of corrected reads is a large amount of data and takes a long time to be evaluated using $Q U A S T$, we only evaluated the first 2000 reads from each dataset with no specific order. Table 7represents error rates in corrected reads and the correction execution time where MiniC.cr refers to the combination of the Minimap2 and Racon correction methods, and Canu.cr refers to reads corrected by Canu. While MiniC.cr correction is faster, Canu result in a lower error rate in corrected reads.

The Effect of Overlap Finding Parameters

As discussed before, the number of $k$-mers to be indexed during the long-read overlap finding step affects the number of discovered overlaps as well as the execution time and peak memory usage. In order to show this effect, we changed the parameters of Minimap2 and processed the same dataset. Table 4 shows the effect of varying $k$ and $w$ on the size of the index (peak memory usage), the number of overlaps found and the time it took to find overlaps. For this evaluation, the 40X coverage long-read data of AJ055 dataset was used.

long-read assembly

Table 5 compares the most recent long-read assembly pipelines which have not been considered in previous reviews. The Mini pipeline was considerably faster than MiniC and Canu as it did not include the most time-consuming step in long-read assembly (read error correction). However, the Mini pipeline achieved high genome coverage and its contiguity is similar to that of MiniC. The Mini pipeline mainly suffered from a high base calling error rate as well as misassemblies. The MiniC pipeline was slower than the Mini pipeline but led to a more accurate base calling in the assembled genome. Canu was the slowest long-read pipeline but resulted in the highest base calling accuracy as well as higher continuity. In addition, Canu requires more memory compared to the Mini pipeline. The memory usage of MiniC is smaller than that of Mini, which is possibly due to fewer false positive overlaps between corrected long-reads (the assembly graph would be smaller). Compared to the short-read assembler (SGA and Spades) as shown in Table 2, long-read assemblies have higher contiguity but at the same time much higher base calling error rates (for Canu only Indels).

Considering the depth of read coverage, the execution time and peak memory usage for all datasets and all programs were lower for $20 \mathrm{X}$ data compared to 40X data. Canu reached almost $100 \%$ genome coverage even with $20 \mathrm{X}$ data. Processing 40X data with Canu resulted in higher contiguity and fewer Indel errors in the assembly. For Mini and MiniC, using higher read coverage improved the genome quality in all aspects. The execution time of Mini and MiniC pipelines were 3.7 and 3.1 times faster (respectively) when processing 20X data compared to $40 \mathrm{X}$ data. This ratio is 1.4 for Canu, which indicates Canu is less sensitive to the read coverage. The memory usage of Mini and $M i n i C$ was almost twice as high for $40 \mathrm{X}$ data compared to $20 \mathrm{X}$ data. This relation is expected as the number of nodes in the overlap graph was equal to the number of reads. Canu memory usage showed less sensitivity to the read coverage.

The effect of genome size on execution time and memory usage of the long-read assembly pipeline is shown in Table6. Apart from the Mini pipeline, which does not include read correction, the execution time of other pipelines almost linearly increased for the longer genome. The memory usage of Canu remained the same for the longer genome. The Mini and MiniC pipelines showed significant increases in the amount of memory used to process the longer genome.

Using a longer genome (SIM25M compared to AJ055) also affect the accuracy of the long-read only pipeline. Important changes are briefly highlighted here (for the complete evaluation metrics for SIM25M refer to https //arashbayat.github.io/asm). The genome coverage of Canu processing low coverage data declined from 
Table 3: Comparing execution time of short-read assembly pipelines when processing different genome size

\begin{tabular}{cc|rrrrrr} 
& & \multicolumn{2}{|c}{ SGA } & \multicolumn{2}{c}{ SOAPdenovo2 } & \multicolumn{2}{c}{ Spades } \\
& Dataset & 40X SR & 20X SR & 40X SR & 20X SR & 40X SR & 20X SR \\
\hline Execution & SIM25M & 3698 & 1718 & 1856 & 1347 & 7775 & 5829 \\
Time (sec) & AJ055 & 633 & 318 & 253 & 94 & 985 & 614 \\
Ratio & & 5.8 & 5.4 & 7.3 & 14.3 & 7.9 & 9.5 \\
\hline Memory & SIM25M & 1905 & 1799 & 8474 & 6760 & 8977 & 6538 \\
Usage (MB) & AJ055 & 1376 & 717 & 5824 & 5125 & 2925 & 1859 \\
Ratio & & 1.4 & 2.5 & 1.5 & 1.3 & 3.1 & 3.5
\end{tabular}

Table 4: The effect of $k$ and $w$ on overlap finding using Minimap2

\begin{tabular}{rc|rrr}
$\mathbf{k}$ & $\mathbf{w}$ & $\begin{array}{r}\text { Number of } \\
\text { Overlaps }\end{array}$ & $\begin{array}{r}\text { Execution } \\
\text { Time }\end{array}$ (sec) & $\begin{array}{r}\text { Memory } \\
\text { (MB) }\end{array}$ \\
\hline $\mathbf{1 0}$ & $\mathbf{1 0}$ & 7750985 & 3453 & 10711 \\
$\mathbf{1 5}$ & $\mathbf{1 0}$ & 229058 & 13.38 & 630 \\
$\mathbf{2 0}$ & $\mathbf{1 0}$ & 181270 & 12.83 & 542 \\
$\mathbf{2 5}$ & $\mathbf{1 0}$ & 163344 & 12.07 & 532 \\
$\mathbf{2 0}$ & $\mathbf{2 0}$ & 162514 & 9.05 & 363 \\
$\mathbf{2 0}$ & $\mathbf{3 0}$ & 145905 & 9.33 & 337
\end{tabular}

Table 5: Comparison of long-read assembly pipeline for the assembly of the AJ055 genome.

\begin{tabular}{l|rrrrrr}
\multicolumn{1}{c|}{ Pipeline Name: } & \multicolumn{2}{c}{ Mini } & \multicolumn{2}{c}{ MiniC } & \multicolumn{2}{c}{ Canu } \\
\multicolumn{1}{c}{ Read Coverage \& Type: } & 20X LR & 40X LR & 20X LR & 40X LR & 20X LR & 40X LR \\
\hline Genome Coverage (\%) & 97.962 & 99.934 & 98.626 & 99.999 & 99.785 & 99.877 \\
\# Contigs & 18 & 2 & 16 & 2 & 9 & 4 \\
N50 & 455694 & 5534010 & 470381 & 5529667 & 3169885 & 5496970 \\
NGA50 & 455694 & 5529303 & 470380 & 5529367 & 3169756 & 5496967 \\
\# Mismatches per 100 kbp & 49.96 & 29.62 & 34.91 & 15.03 & 6.28 & 0.27 \\
\# Indels per 100 kbp & 726.07 & 466.43 & 639.88 & 330.97 & 165.41 & 34.76 \\
\# Local Misassemblies & 4 & 5 & 2 & 1 & 1 & 0 \\
Execution Time (sec) & 70 & 260 & 548 & 1800 & 4070 & 5808 \\
Memory Usage (MB) & 1026 & 2488 & 1019 & 2103 & 3656 & 4994
\end{tabular}

Table 6: The effect of genome size on execution time and memory usage of long-read assembly pipeline

\begin{tabular}{|c|c|c|c|c|c|c|c|}
\hline & & \multicolumn{2}{|c|}{ Mini } & \multicolumn{2}{|c|}{ MiniC } & \multicolumn{2}{|c|}{ Canu } \\
\hline & & $20 \times \mathrm{LF}$ & $40 \times$ LR & $20 \times$ LR & $40 \times \mathrm{LR}$ & $20 \times$ LR & 40X LR \\
\hline Execution Time (sec) & SIM25M & 26 & 561 & 3227 & 13984 & 18906 & 33873 \\
\hline Ratio & AJ055 & 7 & 260 & 548 & 1800 & 4070 & 5808 \\
\hline Ratıo & & 3. & 2.2 & 5.9 & 7.8 & 4.6 & 5.8 \\
\hline Memory Usage (MB) & SIM25M & 426 & $\begin{array}{l}7690 \\
2488\end{array}$ & 4233 & 7725 & 4052 & 5067 \\
\hline Ratio & & $\begin{array}{r}102 \\
4 .\end{array}$ & $\begin{array}{r}2488 \\
3.1\end{array}$ & $\begin{array}{r}1019 \\
4.2\end{array}$ & $\begin{array}{r}2103 \\
3.7\end{array}$ & $\begin{array}{r}656 \\
1.1\end{array}$ & $\begin{array}{r}4994 \\
1.0\end{array}$ \\
\hline
\end{tabular}


99.8\% (for AJ055) to about $85.6 \%$ (for SIM25M). Processing low and high coverage data respectively, the N50 of Canu dropped from 3169885 and 5496970 (for AJ055) to 109830 and 556356 (for SIM25M). The N50 of $M$ iniC pipeline drops from 5529667 (for AJ055) to 2347005 (for SIM25M) processing high coverage data and increased from 470381 (for AJ055) to 1073552 (for SIM25M) when processing low coverage data.

\subsection{Hybrid assembly}

\section{long-read error correction using short-read}

Table 7 compares the Color-Map hybrid correction method with other long-read-only correction methods. Color-Map was much better in correcting Indels mainly due to the low rate of Indels in high-confidence shortreads. However, the correction execution time was significantly larger than a complete long-read-only assembly pipeline. We also assembled a genome using Canu from corrected reads produced by Color-Map. In this case, we inputted corrected reads as pacbio-corrected to Canu, such that Canu did not execute the correction step for the input reads. As shown in Table 8 . while the resulting genome has a lower Indel error rate compared to the Canu pipeline, it lacks contiguity and genome coverage possibly due to the timing applied by Color-Map.

Polishing long-read Assembly using short-read

We evaluated the effect of the Pilon genome polisher on the output of the Canu and the Mini pipelines. We also considered the evaluation of iterative genome polishing using Pilon. In Table 9 p1, p2 and p3 respectively refer to the first, second and third round of polishing with Pilon. The result shows that genome polishing with Pilon can improve base calling accuracy and reduce misassemblies. For the Mini pipeline, which has a higher base calling error rate, the second round of polishing further improved the genome quality. However, the third round of polishing seemed to be less effective and did not provide much improvement. This table provides execution times for one round of polishing with Pilon and does not include genome assembly time. Polishing high-quality assemblies such as Canu takes less time since Pilon exerts less effort in correcting genomes. The execution time of the second and third rounds of polishing of the Mini pipeline also suggest that the time taken for polishing depends on the quality of the input genome.

Other Hybrid Assembly Approach

Table 10 compares the Spades pipeline with and without using long-reads. long-reads are mainly used to scaffold contigs from a short-read assembly. The use of long-read in the Spades pipeline significantly increased contig size while having no effect on the error rate. However, integration of long-read in the Spades pipeline increased execution time. Using long-read also reduced the number of misassemblies.

We used Metassembler to merge three of the best assemblies we obtained, as shown in Table 11. In our evaluation, Metassembler does not lead to an improvement and its output is as accurate as one of the input assemblies (the one obtained with Canu). We did not test Metassembler with low-quality assemblies or large than tree assemblies. Perhaps Metassembler better fit such cases.

\section{CONCLUSION}

As mentioned in Section 1 and based on the results provided in this review, it is difficult to name a pipeline that can be considered best for all sort and volume of data and to improve all aspect of accuracy and performance. Based on the type and volume of data as well as the purpose of assembly, one should choose the pipeline that best suits the application. This review provides guidelines that can be summarised as follows:

- If high coverage long-read data are available, assembling genomes using long-read pipelines seems to be a reasonable method. Polishing genomes once or twice with short-read additionally improves the quality.

- If low coverage long-reads are available, they can be used for the Spades hybrid pipeline to improve contig length in the short-read assembly.

- If only short-reads are available, Spades results in higher genome coverage and contiguity while $S G A$ results in an accurate base calling.

- In case speed is matter one can use SOAPdenovo2 for short-reads and Mini for long-reads. Also, the correction of long-read using short-read is not recommended as it is quite time-consuming.

\section{ACKNOWLEDGEMENTS}

Marc R Wilkins acknowledges funding from Bioplatforms Australia under the federal government NCRIS scheme. Marc R Wilkins also acknowledges funding from the New South Wales State Government RAAP scheme.

\section{References}

[1] Kin Fai Au, Jason G Underwood, Lawrence Lee, and Wing Hung Wong. Improving pacbio long read accuracy by short read alignment. PloS one, 2012 .

[2] Anton Bankevich, Sergey Nurk, Dmitry Antipov, Alexey A Gurevich, Mikhail Dvorkin, Alexander S Kulikov, Valery M Lesin, Sergey I Nikolenko, Son Pham, Andrey D Prjibelski, et al. Spades: a new genome assembly algorithm and its applications to single-cell sequencing. Journal of computational biology, 2012.

[3] Ergude Bao and Lingxiao Lan. Halc: High throughput algorithm for long read error correction. BMC bioinformatics, 2017. 
Table 7: Comparison of correction algorithm for the assembly of the AJ055 genome.

\begin{tabular}{l|rrrrr}
$\begin{array}{c}\text { Pipeline Name: } \\
\text { Read Coverage \& Type: }\end{array}$ & $\begin{array}{r}\text { MiniC.cr } \\
\text { 20X LR }\end{array}$ & $\begin{array}{c}\text { MiniC.cr } \\
\text { 40X LR }\end{array}$ & $\begin{array}{r}\text { Canu.cr } \\
\text { 20X LR }\end{array}$ & $\begin{array}{c}\text { Canu.cr } \\
\text { 40X LR }\end{array}$ & $\begin{array}{r}\text { Colormap.cr } \\
\text { 20X LR + 40X SR }\end{array}$ \\
\hline \# Mismatches per 100 kbp & 134.01 & 109.27 & 95.67 & 49.78 & 90.27 \\
\# Indels per 100 kbp & 1254.47 & 1032.45 & 705.65 & 371.26 & 98.9 \\
Execution Time (sec) & 402 & 1630 & 1804 & 3218 & 21239
\end{tabular}

Table 8: Comparison of assembly with hybrid and non-hybrid correction for the assembly of the AJ055 genome.

\begin{tabular}{|c|c|c|}
\hline $\begin{array}{c}\text { Pipeline Name: } \\
\text { Read Coverage } \\
\text { \& Type: }\end{array}$ & $\begin{array}{c}\text { Canu } \\
\text { 20X LR }\end{array}$ & $\begin{array}{l}\text { Colormap } \\
\text { 20X LR + } \\
\text { 40X SR }\end{array}$ \\
\hline Genome Coverage (\%) & 99.785 & 97.625 \\
\hline \# Contigs & 9 & 73 \\
\hline N50 & 3169885 & 218907 \\
\hline NGA50 & 3169756 & 305685 \\
\hline \# Mismatcl & 6.28 & 24.7 \\
\hline \# 1 & 165.41 & 8.04 \\
\hline \# 1 & 1 & 12 \\
\hline Ex & 4070 & 21279 \\
\hline Memory Usage (MB) & 3656 & 5649 \\
\hline
\end{tabular}

Table 9: The effect of (iterative) polishing for the assembly of the AJ055 genome.

\begin{tabular}{|c|c|c|c|c|c|c|}
\hline $\begin{array}{c}\text { Pipeline Name: } \\
\text { Read Coverage } \\
\text { \& Type: }\end{array}$ & $\begin{array}{c}\text { Canu } \\
40 \times \text { LR }\end{array}$ & $\begin{array}{l}\text { Canu.p1 } \\
\text { 40X LR }+ \\
\text { 40X SR }\end{array}$ & $\begin{array}{c}\text { Mini } \\
\text { 40X LR }\end{array}$ & $\begin{array}{l}\text { Mini.p1 } \\
\text { 40X LR + } \\
\text { 40X SR }\end{array}$ & $\begin{array}{l}\text { Mini.p2 } \\
\text { 40X LR }+ \\
\text { 40X SR }\end{array}$ & $\begin{array}{l}\text { Mini.p3 } \\
\text { 40X LR }+ \\
\text { 40X SR }\end{array}$ \\
\hline Genome Coverage (\%) & 99.877 & 99.877 & 99.934 & 99.939 & 99.939 & 99.939 \\
\hline \# Contigs & & & & 5 & 5 & 2 \\
\hline N50 & 5496970 & 5497403 & 5534010 & 5505273 & 5505584 & 5505476 \\
\hline NGA50 & 5496967 & 5497400 & 5529303 & 5500863 & 5501187 & 5501107 \\
\hline \# Mismatches per $100 \mathrm{kbp}$ & 0.27 & 0 & 29.62 & 1.04 & 0.69 & 0.8 \\
\hline \# Indels per $100 \mathrm{kbp}$ & 34.76 & 2.58 & 466.43 & 55.57 & 8.05 & 7.82 \\
\hline \# Local Misassemblies & & 0 & 5 & 2 & 2 & 1 \\
\hline Execution Time (sec) & 5808 & 414 & 260 & 2506 & 2301 & 1215 \\
\hline Memory Usage (MB) & 4944 & 9206 & 2488 & 6874 & 6350 & 5859 \\
\hline
\end{tabular}

Table 10: Comparison of Spades with and without long-reads for the assembly of the AJ055 genome.

\begin{tabular}{l|rr}
\multicolumn{1}{c|}{$\begin{array}{r}\text { Pipeline Name: } \\
\text { Read Coverage } \\
\text { \& Type: }\end{array}$} & Spades & \multicolumn{2}{c}{$\begin{array}{l}\text { SpadesHybrid } \\
\text { 40X SR + } \\
\text { 20X LR }\end{array}$} \\
\hline Genome Coverage (\%) & 99.131 & 99.882 \\
\# Contigs & 64 & 16 \\
N50 & 226024 & 1571477 \\
NGA50 & 226024 & 1571477 \\
\# Mismatches per 100 kbp & 5.22 & 5.57 \\
\# Indels per 100 kbp & 1.45 & 1.75 \\
\# Local Misassemblies & 11 & 1 \\
Execution Time (sec) & 1074 & 2033 \\
Memory Usage (MB) & 2925 & 2925
\end{tabular}

Table 11: Merging three high quality genome with Metassembler for the assembly of the AJ055 genome.

\begin{tabular}{|c|c|c|c|c|}
\hline Pipelin & SGA & Canu & SpadesHybrid & Metassembl \\
\hline Read Coverage \& Type: & $40 \times \mathrm{SR}$ & $40 \times$ LR & $40 X S R+20 X L R$ & $40 X S R+40 X L R$ \\
\hline Genome Coverage (\%) & 97.757 & 99.877 & 99.882 & 99.877 \\
\hline \# Contigs & 1370 & 4 & 16 & 4 \\
\hline N50 & 6394 & 5496970 & 1571477 & 5496970 \\
\hline NGA50 & 6296 & 5496967 & 1571477 & 5496967 \\
\hline \# Mismatches per $100 \mathrm{kbp}$ & 0.06 & 0.27 & 5.57 & 0.27 \\
\hline \# Indels per $100 \mathrm{kbp}$ & 0.59 & 34.76 & 1.75 & 34.76 \\
\hline \# Local Misassemblies & 0 & 0 & 1 & 0 \\
\hline Execution Time (sec) & 663 & 5808 & 2033 & 20535 \\
\hline Memory Usage (MB) & 1376 & 4944 & 2925 & 394 \\
\hline
\end{tabular}


[4] David R Bentley, Shankar Balasubramanian, Harold P Swerdlow, Geoffrey P Smith, John Milton, Clive G Brown, Kevin P Hall, Dirk J Evers, Colin L Barnes, Helen R Bignell, et al. Accurate whole human genome sequencing using reversible terminator chemistry. nature, 2008.

[5] Konstantin Berlin, Sergey Koren, Chen-Shan Chin, James P Drake, Jane M Landolin, and Adam M Phillippy. Assembling large genomes with single-molecule sequencing and localitysensitive hashing. Nature biotechnology, 2015.

[6] bioplatforms. sistant sepsis pathogens. data.bioplatforms.com/organization/about/bpasepsis, 2018.

[7] Marten Boetzer and Walter Pirovano. Toward almost closed genomes with gapfiller. Genome biology, 2012.

[8] Marten Boetzer and Walter Pirovano. Sspacelongread: scaffolding bacterial draft genomes using long read sequence information. BMC bioinformatics, 2014.

[9] Keith R Bradnam, Joseph N Fass, Anton Alexandrov, Paul Baranay, Michael Bechner, Inanç Birol, Sébastien Boisvert, Jarrod A Chapman, Guillaume Chapuis, Rayan Chikhi, et al. Assemblathon 2: evaluating de novo methods of genome assembly in three vertebrate species. GigaScience, 2013.

[10] Andrei Z Broder. On the resemblance and containment of documents. Compression and Complexity of Sequences 1997. Proceedings, 1997.

[11] Chen-Shan Chin, David H Alexander, Patrick Marks, Aaron A Klammer, James Drake, Cheryl Heiner, Alicia Clum, Alex Copeland, John Huddleston, Evan E Eichler, et al. Nonhybrid, finished microbial genome assemblies from long-read smrt sequencing data. Nature methods, 2013.

[12] Chen-Shan Chin, Paul Peluso, Fritz J Sedlazeck, Maria Nattestad, Gregory T Concepcion, Alicia Clum, Christopher Dunn, Ronan O'Malley, Rosa Figueroa-Balderas, Abraham Morales-Cruz, et al. Phased diploid genome assembly with singlemolecule real-time sequencing. Nature methods, 2016.

[13] Gennady Denisov, Brian Walenz, Aaron L Halpern, Jason Miller, Nelson Axelrod, Samuel Levy, and Granger Sutton. Consensus generation and variant detection by celera assembler. Bioinformatics, 2008.

[14] John Eid, Adrian Fehr, Jeremy Gray, Khai Luong, John Lyle, Geoff Otto, Paul Peluso, David Rank, Primo Baybayan, Brad Bettman, et al. Real-time dna sequencing from single polymerase molecules. Science, 2009.
[15] Paolo Ferragina and Giovanni Manzini. An experimental study of an opportunistic index. Proceedings of the twelfth annual ACM-SIAM symposium on Discrete algorithms, 2001.

[16] Alexey Gurevich, Vladislav Saveliev, Nikolay Vyahhi, and Glenn Tesler. Quast: quality assessment tool for genome assemblies. Bioinformatics, 2013.

[17] Ehsan Haghshenas, Faraz Hach, S Cenk Sahinalp, and Cedric Chauve. Colormap: Correcting long reads by mapping short reads. Bioinformatics, 2016.

[18] Ruifeng Hu, Guibo Sun, and Xiaobo Sun. Lscplus: a fast solution for improving long read accuracy by short read alignment. BMC bioinformatics, 2016.

[19] Weichun Huang, Leping Li, Jason R Myers, and Gabor T Marth. ART: a next-generation sequencing read simulator. Bioinformatics (Oxford, England), 2012.

[20] Miten Jain, Hugh E Olsen, Benedict Paten, and Mark Akeson. The oxford nanopore minion: delivery of nanopore sequencing to the genomics community. Genome biology, 2016.

[21] Juhana I Kammonen, Olli-Pekka Smolander, Lars Paulin, Pedro AB Pereira, Pia Laine, Patrik Koskinen, Jukka Jernvall, and Petri Auvinen. gapfinisher: a reliable gap filling pipeline for sspacelongread scaffolder output. PeerJ PrePrints, 2017.

[22] David R Kelley, Michael C Schatz, and Steven L Salzberg. Quake: quality-aware detection and correction of sequencing errors. Genome biology, 2010 .

[23] Jan O Korbel, Alexander Eckehart Urban, Jason P Affourtit, Brian Godwin, Fabian Grubert, Jan Fredrik Simons, Philip M Kim, Dean Palejev, Nicholas J Carriero, Lei Du, et al. Paired-end mapping reveals extensive structural variation in the human genome. Science, 2007.

[24] Sergey Koren, Brian P Walenz, Konstantin Berlin, Jason R Miller, Nicholas H Bergman, and Adam M Phillippy. Canu: scalable and accurate long-read assembly via adaptive k-mer weighting and repeat separation. Genome research, 2017.

[25] Heng Li. Klib library. attractivechaos.github.io/klib, 2011.

[26] Heng Li. Aligning sequence reads, clone sequences and assembly contigs with BWA-MEM. arXiv preprint arXiv:1303.3997, 2013.

[27] Heng Li. Minimap and miniasm: fast mapping and de novo assembly for noisy long sequences. Bioinformatics, 2016.

[28] Heng Li. Minimap2: pairwise alignment for nucleotide sequences. Bioinformatics, 2018. 
[29] Zhenyu Li, Yanxiang Chen, Desheng Mu, Jianying Yuan, Yujian Shi, Hao Zhang, Jun Gan, Nan Li, Xuesong $\mathrm{Hu}$, Binghang Liu, Bicheng Yang, and Wei Fan. Comparison of the two major classes of assembly algorithms: overlap layout consensus and de bruijn graph. Briefings in Functional Genomics, 2012.

[30] Ruibang Luo, Binghang Liu, Yinlong Xie, Zhenyu Li, Weihua Huang, Jianying Yuan, Guangzhu He, Yanxiang Chen, Qi Pan, Yunjie Liu, et al. Soapdenovo2: an empirically improved memory-efficient short-read de novo assembler. Gigascience, 2012.

[31] Michael L Metzker. Sequencing technologiesthe next generation. Nature reviews genetics, 2010.

[32] Jason R Miller, Arthur L Delcher, Sergey Koren, Eli Venter, Brian P Walenz, Anushka Brownley, Justin Johnson, Kelvin Li, Clark Mobarry, and Granger Sutton. Aggressive assembly of pyrosequencing reads with mates. Bioinformatics, 2008.

[33] Eugene W Myers. Ano (nd) difference algorithm and its variations. Algorithmica, 1986.

[34] Eugene W Myers. The fragment assembly string graph. Bioinformatics, 2005.

[35] Eugene W Myers, Granger G Sutton, Art L Delcher, Ian M Dew, Dan P Fasulo, Michael J Flanigan, Saul A Kravitz, Clark M Mobarry, Knut HJ Reinert, Karin A Remington, et al. A whole-genome assembly of drosophila. Science, 2000.

[36] Gene Myers. A fast bit-vector algorithm for approximate string matching based on dynamic programming. Journal of the ACM, 1999.

[37] Giuseppe Narzisi and Bud Mishra. Comparing de novo genome assembly: the long and short of it. PloS one, 2011.

[38] Vicki Pandey, Robert C Nutter, and Ellen Prediger. Applied biosystems solid system: ligationbased sequencing. Next Generation Genome Sequencing: Towards Personalized Medicine, 2008.

[39] Pavel A Pevzner, Haixu Tang, and Michael S Waterman. An eulerian path approach to dna fragment assembly. Proceedings of the National Academy of Sciences, 2001.

[40] Michael Roberts, Wayne Hayes, Brian R Hunt, Stephen M Mount, and James A Yorke. Reducing storage requirements for biological sequence comparison. Bioinformatics, 2004.

[41] Leena Salmela and Eric Rivals. Lordec: accurate and efficient long read error correction. Bioinformatics, 2014.

[42] Jared T Simpson and Richard Durbin. Efficient construction of an assembly string graph using the fm-index. Bioinformatics, 2010.
[43] Jared T Simpson and Richard Durbin. Efficient de novo assembly of large genomes using compressed data structures. Genome research, 2012.

[44] Jared T Simpson and Richard Durbin. Efficient de novo assembly of large genomes using compressed data structures. Genome research, 2012.

[45] T.F. Smith and M.S. Waterman. Identification of common molecular subsequences. Journal of Molecular Biology, 1981.

[46] Jang-il Sohn and Jin-Wu Nam. The present and future of de novo whole-genome assembly. Briefings in bioinformatics, 2016.

[47] Martin Sosic and Mile Sikic. Edlib: A C/C++ library for fast, exact sequence alignment using edit distance. bioRxiv, 2016.

[48] Bianca K Stöcker, Johannes Köster, and Sven Rahmann. Simlord: Simulation of long read data. Bioinformatics, 2016.

[49] Robert Vaser, Ivan Sović, Niranjan Nagarajan, and Mile Šikić. Fast and accurate de novo genome assembly from long uncorrected reads. Genome research, 2017.

[50] Sarah Vergult, Ellen Van Binsbergen, Tom Sante, Silke Nowak, Olivier Vanakker, Kathleen Claes, Bruce Poppe, Nathalie Van der Aa, Markus J Van Roosmalen, Karen Duran, et al. Mate pair sequencing for the detection of chromosomal aberrations in patients with intellectual disability and congenital malformations. European Journal of Human Genetics, 2014.

[51] Bruce J Walker, Thomas Abeel, Terrance Shea, Margaret Priest, Amr Abouelliel, Sharadha Sakthikumar, Christina A Cuomo, Qiandong Zeng, Jennifer Wortman, Sarah K Young, et al. Pilon: an integrated tool for comprehensive microbial variant detection and genome assembly improvement. PloS one, 2014.

[52] Alejandro Hernandez Wences and Michael C Schatz. Metassembler: merging and optimizing de novo genome assemblies. Genome biology, 2015.

[53] Matei Zaharia, William J Bolosky, Kristal Curtis, Armando Fox, David Patterson, Scott Shenker, Ion Stoica, Richard M Karp, and Taylor Sittler. Faster and More Accurate Sequence Alignment with SNAP. arXiv, 2011.

[54] Daniel R Zerbino and Ewan Birney. Velvet: algorithms for de novo short read assembly using de bruijn graphs. Genome research, 2008.

[55] Aleksey V Zimin, Guillaume Marçais, Daniela Puiu, Michael Roberts, Steven L Salzberg, and James A Yorke. The masurca genome assembler. Bioinformatics, 2013. 